ISSN 2073-8994

www.mdpi.com/journal/symmetry

\title{
Commentary
}

\section{Symmetry at the Foundation of Science and Nature}

\author{
Joe Rosen $\#, \dagger$ \\ Adjunct Professor, The George Washington University, Washington DC, USA; \\ E-mail: joerosen@mailaps.org \\ \# Private Address: 338 New Mark Esplanade, Rockville MD 20850, USA \\ ${ }^{\dagger}$ Retired from Tel Aviv University, Tel Aviv, Israel
}

Received: 1 May 2009 / Accepted: 4 June 2009 / Published: 5 June 2009

\begin{abstract}
This article demonstrates that science is founded on symmetry and that Nature must have symmetry at its foundation. Full details are given in the book: Rosen, J. Symmetry Rules: How Science and Nature Are Founded on Symmetry; Springer-Verlag: Berlin, Germany, 2008.
\end{abstract}

Keywords: symmetry; science; nature

\section{Introduction}

Science rests firmly on the foundation of symmetry. By means of science, we view Nature through symmetry spectacles and understand nature in the language of symmetry. Might we then claim that science is symmetry or even that nature is symmetry? This article presents a brief review of ideas presented in detail in Rosen [1].

Symmetry manifests itself at the foundation of science in (at least) seven forms. These are:

1. reproducibility,

2. predictability,

3. reduction,

4. symmetry of evolution,

5. symmetry of states,

6. gauge symmetry,

7. symmetry at the foundation of quantum theory.

The first three are obvious, essential components of the foundation of science. Science would not be science without reproducibility and predictability, and science has very successfully operated by 
reduction (although holistic considerations are becoming more and more necessary). That these three are indeed symmetries is discussed in the following. The last four are most commonly viewed as lying at the foundation of Physics. However, Physics underlies all the other sciences, so these symmetries lie also at the foundation of science.

Let us start with a reminder of what symmetry [2] is:

Symmetry is immunity to a possible change.

Note the two essential components of symmetry:

1. Possibility of change. It must be possible to perform a change, although the change does not actually have to be performed.

2. Immunity. Some aspect of the situation would remain unchanged, if the change were performed.

Now consider each of the seven symmetries enumerated earlier.

\section{Reproducibility}

Reproducibility is the replicability of experiments in the same laboratory and in other laboratories. When certain changes (such as in location, time, velocity, or apparatus composition) are imposed on the experiment, the result of the changed experiment is the similarly changed result of the original experiment. This is symmetry. Its two components are:

1. Possibility of change. Changes belonging to the set associated with reproducibility can be performed on the experiment and its result.

2. Immunity. The changed result remains the actual result of the changed experiment.

\section{Predictability}

Predictability means that among the phenomena investigated, order can be found, from which laws can be formulated, predicting the results of new experiments. In a predictable situation there exists a well confirmed relation $R$ between experimental input and experimental result:

$$
\text { Input } \stackrel{R}{\longrightarrow} \text { Result }
$$

The relation $R$ serves to predict the result of new experiments. This, too, is symmetry, with the components:

1. Possibility of change. The input to the experiment can be changed.

2. Immunity. The experimental result maintains the same relation $R$ with the experimental input. 


\section{Reduction}

In order to make Nature amenable to investigation, science has always resorted to reduction and still does so with great success (note, however, that quantum theory and cosmology are encouraging a more holistic approach). Reduction is the literal or figurative separation of Nature into parts that can be individually understood, parts that exhibit order and law and are explainable regardless and independently of what is going on in the rest of Nature. This is symmetry. The two components are:

1. Possibility of change. It is possible to make changes in the rest of Nature.

2. Immunity. These changes do not affect important aspects of the part of Nature that can be individually understood.

Reduction of Nature can be carried out in different ways. Here are three ways reduction is commonly carried out in science.

\section{1) Reduction to observer and observed}

The reduction of Nature to observer and observed is based on this assumption, or working hypothesis: The effect of our observations on what we observe is sufficiently weak or can be made so, that what we actually observe well reflects what would occur without our observation and the understanding we reach under this assumption is relevant to the actual situation. Quantum theory imposes intrinsic limits on the validity of this reduction and in classical situations there might exist practical limits. But when observer-observed reduction is valid, it is symmetry, one aspect of which is expressed in the two components:

1. Possibility of change. We can change our observational activities.

2. Immunity. The behavior of the rest of Nature is unaffected by and independent of such changes.

This symmetry allows the compilation of objective, observer-independent data about Nature. Objectivity, too, is symmetry, with the components:

1. Possibility of change. Observers can be changed.

2. Immunity. The validity of the data is independent of the observer.

\section{2) Reduction to quasi-isolated system and environment}

A perfectly isolated system cannot be observed, does not interact with the rest of the Universe, and thus does not form part of Nature (as far is we scientists are concerned). A quasi-isolated system is one that is sufficiently isolated for some purpose. Quantum theory and the Mach principle, if valid, set limits on the isolability of systems, and for classical systems even quasi-isolation might not be possible. Science reduces Nature to quasi-isolated system and environment, when possible, in order to study the system independently of an uncontrollable environment. The separation of Nature into quasiisolated system and environment will be a reduction, if, in spite of the system's imperfect isolation, there are aspects of the system that are nevertheless unaffected by its environment, at least to a sufficient extent. This is symmetry. One side of this symmetry is expressed as follows: 
1. Possibility of change. Changes can be made in the environment of a quasi-isolated system.

2. Immunity. A quasi-isolated system possesses aspects that are not affected by certain such changes.

\section{3) Reduction to initial state and evolution}

This reduction is the conceptual separation of natural processes into initial state and evolution. When it is done successfully for quasi-isolated systems, laws are discovered that, for any initial state of the system, give the state of the system that evolves from it at any subsequent time. In other words, initial state and law of evolution are independent of each other. One symmetry of this reduction has these components:

1. Possibility of change. The state of a system, as an initial state, can be changed.

2. Immunity. The law of evolution for the system is the same no matter what the system's initial state.

This reduction runs into trouble for the Universe as a whole.

\section{Symmetry of Evolution}

Symmetry of evolution of quasi-isolated systems is an important and useful manifestation of symmetry in Physics. This symmetry has to do with transformations that map physical processes, or evolutions, into physical processes and hypothetical unphysical processes into unphysical ones. The two components are:

1. Possibility of change. A process in a quasi-isolated system can be transformed to another process.

2. Immunity. A physical process retains its physicality, while a hypothetical unphysical process remains unphysical.

\section{Symmetry of States}

Transformations within a quasi-isolated system might map states to image states that are indistinguishable from their object states within the system. This symmetry has the components:

1. Possibility of change. A state of a quasi-isolated system can be transformed to another state of the system.

2. Immunity. The object and image states are indistinguishable within the system.

\section{Gauge Symmetry}

This symmetry is a very particular type of symmetry of evolution, sufficiently special and important to be listed in its own separate category. Very roughly, gauge symmetry involves groups of certain continuous transformations with space-time-dependent parameters, called gauge transformations (the transformations involved in symmetry of evolution listed in the Introduction as number 4 and 
presented above in Section 5 are generally understood to have space-time-independent parameters). These transformations relate inertial processes, or evolutions, and dynamic ones in such a way that if a process is physical or hypothetically unphysical, evolutions related to it by such gauge transformations are also physical or unphysical, respectively. In quantum field theory gauge symmetry severely constrains the dynamics. The two components of this symmetry are:

1. Possibility of change. A process in a quasi-isolated system can be transformed to another process by any gauge transformation of certain kinds.

2. Immunity. A physical process retains its physicality, while a hypothetical unphysical process remains unphysical. In particular, a dynamic process related to a physical inertial process by such a gauge transformation is also physical.

Please refer to Rosen [1] for much more detail.

\section{Symmetry at the Foundation of Quantum Theory}

I have found ten manifestations of symmetry underlying quantum theory and present them in the following along with very brief descriptions of the symmetry or symmetries involved. See Rosen [1] for the details.

\subsection{Association of a Hilbert space with a physical system}

(1) The physical significance of the association is immune to possible change of representation of the Hilbert space. (2) The correspondence of a vector (or unit vector) of a system's Hilbert space to a state of the system is immune to multiplication of the vector (unit vector) by a complex number (by a phase factor). (3) The physical properties and evolution determined by quantum theory are immune to possible changes in aspects of states that are not comprehended by vectors of the system's Hilbert space.

\subsection{Correspondence of observables to Hermitian operators}

(1) This correspondence is immune to possible changes of representation of the operator. (2) This correspondence is immune to possible changes in the process of measurement of an observable. (3) The postulate that the possible values of an observable for a given system are eigenvalues of the corresponding Hermitian operator is immune to the actual measurement result (which is thus constrained to the operator's eigenvalue spectrum). (4) The latter holds true in particular for the same measurement procedure applied to the same state.

\subsection{Complete set of compatible observables}

(1) The physical properties and evolution determined by quantum theory are immune to possible change in the way a state is specified, and thus also to possible change in the choice of complete set of compatible observables. (2) The physical significance of the association of a Hilbert space with a system is immune to possible change in the choice of complete set of compatible observables. 


\subsection{Heisenberg commutation relations}

For a quantized classical system described in terms of a set of canonical variables, the Heisenberg commutation relations for the Hermitian operators corresponding to the canonical variables are immune to possible canonical transformation of those variables.

\subsection{Operators for canonical variables}

For a quantized classical system described in terms of a set of canonical variables, the physical significance of a Hermitian operator is immune to possible change in its form entailed by canonical transformation of the canonical variables.

\subsection{A measurement result is an eigenvalue}

The effect of a measurement - the indeterministic projection of a system's state onto one of the eigenstates of the measured observable - is immune to possible change of the state of the system.

\subsection{Expectation values and probabilities}

Calculated expectation values and probabilities are immune to the choice of vector from the ray (or unit ray) corresponding to a state of the system.

\subsection{The Hamiltonian operator}

The Hamiltonian's property of being the generator of deterministic evolution is immune to possible change of the state of the evolving system.

\subsection{Planck's constant as a parameter}

The mathematical formalism of quantum theory is immune to possible change of the actual value of $h$.

\subsection{The correspondence principle}

(1) The validity of the general correspondence principle - that any physical theory more general than classical theory must be consistent with classical theory in the latter's domain of validity-is immune to possible change of the more general physical theory to which it is applied. (2) The particular correspondence principle for quantum theory, which generally involves the limit $h \rightarrow 0$, shares this symmetry, in that it is immune to possible change of the actual value of $h$.

\section{Science and Nature}

Thus, symmetry composes most, if not all, of the foundation of Science. Some even say Science $i s$ symmetry. I am sympathetic with this viewpoint. Science presents us with a picture of Nature that is extremely symmetric, i.e., a view through symmetry spectacles, so to speak. Furthermore, Science gives us an understanding of Nature that is formulated in the language of symmetry. Since Science is so successful, we can safely conclude that Nature in fact possesses much symmetry at its foundation. 
Is Nature all about symmetry? I do not think we are presently in a position to make such a claim. We know there is still much to learn about Nature. But I would not be surprised if, as we attain further understanding of Nature's deeper strata, we find that symmetry underlies Nature-mostly, if not totally. Perhaps, indeed, Nature is symmetry.

\section{References}

1. Rosen, J. Symmetry Rules: How Science and Nature Are Founded on Symmetry; Springer-Verlag: Berlin, Germany, 2008.

2. Rosen, J. The Symmetry Principle. Entropy 2005, 7, 308-313; doi:10.3390/e7040308.

(C) 2009 by the authors; licensee Molecular Diversity Preservation International, Basel, Switzerland. This article is an open-access article distributed under the terms and conditions of the Creative Commons Attribution license (http://creativecommons.org/licenses/by/3.0/). 